(44.8\%) described that they had never used condoms. In 2013, 838 men were attended, 84 (10.02\%) were diagnosed with HIV. 5 patients (5.9\%) reported having between 1 and 3 years of complete school education, $32(38 \%)$ had between 4 and 7 years of school education, and $36(42.8 \%)$ between 8 and 11 years. $45(53.6 \%)$ described having experienced previous STDs. 7 patients (8.3\%) reported effective condom use, but 33 men (39.3\%) have never used condoms.

Conclusion It has been analysed that, after 10 years, an infection growth has taken place among individuals with a higher educational level, an increase of concomitant or past STDs in the previous year before the test, corroborating with the reduction in condom use. This study shows the need of the health professional assistance regarding, not only the pre-and post-test counselling, but also the educational activities within the communities in order to carry out awareness-raising strategies and guidelines aimed at STD/HIV prevention among male individuals.

\section{P3.212 ANTIBIOTIC RESISTANCE DETECTION IS ESSENTIAL FOR GONORRHOEA POINT-OF-CARE TESTING: A MATHEMATICAL MODELLING STUDY}

${ }^{1}$ Stephanie M Fingerhuth, ${ }^{2}$ Nicola Low, ${ }^{1}$ Sebastian Bonhoeffer, ${ }^{2}$ Christian L Althaus. ${ }^{1}$ ETH Zurich, Zurich, Switzerland; ${ }^{2}$ University of Bern, Bern, Switzerland

\subsection{6/sextrans-2017-053264.447}

Introduction Antibiotic resistance threatens to make Neisseria gonorrhoeae untreatable. Point-of-care tests (POC) that detect antimicrobial resistance (AMR) would allow individually tailored treatment. But rapid access to test results might lead to more treatment overall, resulting in higher resistance levels. We investigated the impact of different clinical pathways for gonorrhoea diagnosis on the spread of AMR gonorrhoea.

Methods We used data about the prevalence and incidence of gonorrhoea in men who have sex with men (MSM) and heterosexual men and women (HMW) to calibrate a mathematical model that describes the transmission of $N$. gonorrhoeae. With this model, we simulated four clinical pathways for the diagnosis and treatment of gonorrhoea: POC test for N. gonorrhoeae with $\mathrm{AMR}$ detection $(\mathrm{POC}+\mathrm{R}), \mathrm{POC}$ without $\mathrm{AMR}$ detection (POC-R), culture with antimicrobial susceptibility testing (culture), and laboratory-based nucleic acid amplification tests without AMR detection (NAAT). We calculated the proportion of resistant infections, the cases averted after 5 years, and compared how fast resistant infections spread in the populations.

Results After 30 years, the proportion of resistant N. gonorrhoeae infections is lowest for POC $+\mathrm{R}$ (median MSM: $0.18 \%$, HMW: $0.12 \%$ ), and increases for culture, NAAT, and POC-R. After 5 years, NAAT leads to a total of 36366 (median MSM) and 1228 (median HMW) observed cases per 100000 persons. $\mathrm{POC}+\mathrm{R}$ results in the largest number of cases averted after 5 years (median MSM: 3,353, HMW: 118 per 100000 persons) compared with NAAT. POC tests with intermediate sensitivity for the detection of AMR slow the spread of resistance more than NAAT. POC tests require very high sensitivity to detect AMR to reduce the spread of AMR more than culture.

Conclusion POC tests with high sensitivity to detect AMR can keep gonorrhoea treatable for longer than either culture or NAAT. POC tests that do not detect AMR reliably should not be introduced because they result in higher levels of empirical treatment for gonorrhoea and accelerate the spread of AMR.

\section{P3.213 A TOOL FOR EVALUATING THE IMPACT OF THE NATIONAL CHLAMYDIA SCREENING PROGRAMME IN ENGLAND: C. TRACHOMATIS ANTIBODY PREVALENCE IN YOUNG WOMEN IN ENGLAND (2007-2015)}

${ }^{1}$ Stephanie Migchelsen, ${ }^{2}$ Gillian Wills, ${ }^{3}$ Paddy Horner, ${ }^{4}$ Ezra Linley, ${ }^{2}$ Eleanor Mcclure, ${ }^{1}$ Kate Soldan, ${ }^{2}$ Myra Mcclure, ${ }^{1}$ Kevin Dunbar, 'Sarah Woodhall. ${ }^{1}$ Public Health England, London, UK; ${ }^{2}$ Imperial College London, London, UK; ${ }^{3}$ University of Bristol, Bristol, UK; ${ }^{4}$ Public Health England, Manchester, UK

\subsection{6/sextrans-2017-053264.448}

Introduction Genital infection with Chlamydia trachomatis (CT) is the most commonly-diagnosed bacterial sexually transmitted infection in England. The National Chlamydia Screening Programme (NCSP) was implemented nationwide in 2008, offering opportunistic CT testing to people under 25. Not all chlamydia infections result in a lasting antibody response, however, monitoring age-specific seroprevalence of antibodies against CT over time may offer insights into the impact of this intervention. We explored trends in seroprevalence from 2007 up to 2015.

Methods Samples were obtained from the PHE Seroepidemiology Unit, which collects unlinked, anonymous, residual sera submitted to laboratories in England for routine investigations. Samples known to come from GUM clinics were excluded. Sera from 2007-2015 from women aged 15-30 $(n=9,798)$ were tested using an indirect IgG ELISA for chlamydia Pgp3 antibody. Women in 2007 had limited exposure to the NCSP, increasing over time. Age-standardised seroprevalence was calculated for 17-24 year-olds using 2015 population data. Samples were classified by the number of years individuals were eligible for the screening programme, based on year of birth.

Results Age-standardised seroprevalence among 17-24 year-olds varied, being highest at 20.3\% (95\% CI 17.2-23.4) in 2007 and lowest at $15.5 \%$ (95\% CI 10.0-20.9) in 2015, although no clear trend was seen. Although incomplete data were available for those with 'limited' and 'high' exposure to the NCSP, age-specific seroprevalence did not vary by exposure to NCSP. Conclusion There was no evidence that age-specific seroprevalence varied by exposure to the NSCP. Interpretation of this is complicated by the potential effects of antibody prevalence waning over time, and being affected by factors such as treatment and re-infection. Other limitations include a high number $(86.2 \%)$ of specimens from 'unknown' source which could have been from GUM clinics. Multi-parameter evidence synthesis models are being developed to explore the use of these data to estimate incidence.

\section{P3.214 TRACKING THE USE AND RE-EMERGENCE OF SEROLOGICAL TECHNIQUES FOR CHLAMYDIA TRACHOMATIS ANTIBODY DETECTION: A SYSTEMATIC REVIEW}

${ }^{1}$ Stephanie Migchelsen, ${ }^{2}$ Sarah C Woodhall, ${ }^{3}$ David Mabey, ${ }^{3}$ Chrissy H Roberts. ${ }^{1}$ Public Health England, London, UK; ${ }^{2}$ Public Health England, Londn, UK; ${ }^{3}$ London School of Hygiene and Tropical Medicine, London, UK

10.1136/sextrans-2017-053264.449 
Introduction The presence of antibodies against Chlamydia trachomatis $(\mathrm{Ct})$ is indicative of previous genital or ocular infection. Serology was introduced in the 1970s to support the diagnosis of pelvic inflammatory disease (PID), but fell out of favour due to its cross-reactivity with Chlamydophila (Chlamydia) pneumoniae bacteria. With the recent development of sensitive and specific assays, as well as the identification of immunogenic Ct antigens developed as recombinant proteins, serology holds the potential to be a useful tool in public health. To date, there has been no summary of the techniques used, their development and their potential usefulness in public and tropical medicine.

Methods We searched PubMed, Cochrane, Lilacs, Scielo, Scopus and Web of Science for articles published on serological techniques and their use in a public health context. Studies were categorised by technique employed, antigen used and antibody detected.

Results A total of 16 studies were included- 5 related to ocular Ct infection and 11 related to genital $\mathrm{Ct}$ infection. The trachoma studies were predominantly based on Tanzanian samples, while the genital studies were based on samples from an array of countries. The studies were heterogeneous in design, assay and antigen used, and immunoglobulin detected. The estimated prevalence of antibodies against $\mathrm{Ct}$ in trachoma studies ranged from $0 \%-62 \%$; from $0 \%-88.9 \%$ in genital studies. For genital Ct infections, serology is commonly used to explore disease sequelae. For ocular Ct infections, serology is explored as a means to monitor elimination efforts.

Conclusion Techniques used to measure the prevalence of antibodies against $\mathrm{Ct}$ have reported increased sensitivity and specificity. There is wide diversity in antigens and assays used and antibodies detected. The practicality of an assay depends on resources available, purpose of the study, and population being studied. There is wide scope for the development and refinement of techniques to increase the value of serology as it relates to development of new techniques, research and public health.

\section{P3.215 CHARACTERISE THE TEMPORAL EVOLUTION OF HIV INCIDENCE AMONG STABLE COUPLES IN SUB-SAHARAN AFRICA}

Susanne Awad, Hiam Chemaitelly, Laith J Abu-Raddad. Weill Cornell Medicine-Qatar, Doha, Qatar

10.1136/sextrans-2017-053264.450

Introduction We quantified and characterised the temporal evolution of the contribution of several types of sexual-partnership HIV statuses to total HIV incidence in representative countries at different HIV epidemic scales in sub-Saharan Africa.

Methods A pair-based mathematical model was constructed to accommodate for movement of individuals between different forms of sexual partnerships and HIV infection statuses. Stable couples (SCs) were divided into sero-concordant negative (SCNCs), sero-discordant (SDCs), and sero-concordant positive couples. Throughout epidemic phases, historical and future trends (1980-2030) of sources of HIV incidence by partnership status were projected based on nationally-representative epidemiologic and demographic data.

Results As the epidemics emerged and peaked, HIV incidence resulting among SDCs increased from $6 \%-32 \%$ to $19 \%-44 \%$ of total HIV incidence, mostly due to transmissions within the SDCs. As the epidemics started their natural and further decline with antiretroviral therapy (ART) scale-up, this proportion declined in intermediate and high HIV-prevalence countries, but increased in low HIV-prevalence countries. As the epidemics emerged and peaked, HIV incidence resulting among SCNCs from extramarital sex declined from 54\%-80\% to $35 \%-73 \%$ of total HIV incidence. As the epidemics started their decline, this proportion increased in intermediate and high HIV-prevalence countries, but declined in low HIV-prevalence countries. HIV incidence resulting among individuals not in SCs was 9\%-29\% of total HIV incidence and was stable throughout the epidemics, but larger with higher HIV prevalence.

Conclusion The contribution of different forms of sexual-partnership HIV statuses to total HIV incidence was dependent on HIV epidemic phase and scale. Throughout the epidemics, more than two thirds of HIV incidence occurred among individuals engaged in SCs. The majority of incidence among SCs was due to extramarital sex. To achieve reductions in HIV incidence, prevention approaches should target both SCs and individuals not in SCs.

\section{P3.216 ASSESSING HPV GENOTYPE PREVALENCE IN INFECTION AND DISEASE IN YOUNG AUSTRALIAN WOMEN FOLLOWING THE INTRODUCTION OF A NATIONAL VACCINATION PROGRAM}

${ }^{1}$ Suzanne Garland, ${ }^{2} \mathrm{AM}$ Cornall, ${ }^{3} \mathrm{JML}$ Brotherton, ${ }^{4} \mathrm{SL}$ Osborne, ${ }^{5} \mathrm{CD}$ Wrede, ${ }^{6} \mathrm{JD}$ Wark ${ }^{3} \mathrm{M}$ Saville, ${ }^{7} \mathrm{~J}$ Pyman, ${ }^{8} \mathrm{~J}$ Tan, ${ }^{9} \mathrm{ET}$ Callegari, ${ }^{3} \mathrm{DM}$ Gertig, ${ }^{10} \mathrm{Y}$ Jayasinghe, ${ }^{11} \mathrm{M}$ Malloy, ${ }^{12} \mathrm{M}$ Pitts, ${ }^{1} \mathrm{SN}$ Tabrizi. ${ }^{1}$ Royal Women's Hospital, Department of Microbiology and Infectious Diseases, Parkville, Australia; ${ }^{2}$ Royal Women's Hospital, Department of Microbiology and Infectious Diseases, and Murdoch, Parkville, Australia; ${ }^{3}$ National HPV Vaccination Program Register, VCS, East Melbourne, Australia; ${ }^{4}$ Royal Women's Hospital, Department of Microbiology and Infectious Diseases, Parkville, and Murdoch, Parkville, Australia; ${ }^{5}$ Royal Women's Hospital, Parkville, Australia; ${ }^{6}$ The University of Melbourne, Parkville, Australia; ${ }^{7}$ Department of Anatomical Pathology, The Royal Women's Hospital, Parkville, Australia; ${ }^{8}$ Department of Obstetrics and Gynaecology, University of Melbourne and Oncology and Dysplasia Unit, R, Parkville, Australia; ${ }^{9}$ The University of Melbourne, Department of Medicine, Royal Melbourne Hospital, Parkville, Australia; ${ }^{10}$ Department of Obstetrics and Gynaecology, University of Melbourne, Department of Gynaecology, Roya, Parkville, Australia; ${ }^{11}$ National Hpv Vaccination Program Register, VCS, Parkville, Australia; ${ }^{12}$ Australian Research Centre in Sexual Health and Society, La Trobe University, Melbourne, Australia

10.1136/sextrans-2017-053264.451

Introduction The National HPV Vaccination Program, utilising the quadrivalent HPV vaccine, was implemented in Australia in 2007. As the first country to introduce this scheme, Australia is ideally placed to identify changes in HPV genoprevalence in a vaccinated population, as well as in cervical intraepithelial neoplasia grade 3 (CIN3) lesions.

Methods The VACCINE (Vaccine Against Cervical Cancer Impact and Effectiveness) study was designed to assess prevalence of vaccine-targeted HPV genotype infections. In substudy A, sexually active Victorian women aged 18-25 years, recruited through targeted social media advertising on Facebook, were asked to complete an online questionnaire and provide a self-collected vaginal swab for HPV DNA genotyping. The National HPV Vaccination Program Register (NHVPR) was utilised to verify self-reported vaccination status. In sub-study B, causal HPV genotypes in 529 CIN3 cases among vaccine-eligible young women were determined using 